\title{
The Initial Growth of Vapour Deposited Gold Films
}

\author{
Thorwald G. Andersson \\ Department of Physics, Chalmers University of Technology, Göteborg, Sweden \\ Gold films are extensively used in the production of both ohmic-and Schottky-type \\ electrical contacts to semiconductors. Although their growth by vapour deposition has \\ been extensively studied, much less is known of the stages which precede than those \\ which follow nucleation. The pre-nucleation stages of the growth of gold films on \\ clean and ordered as well as contaminated surfaces of silicon and gallium arsenide \\ $(G a A s)$ are the subject of the present review. It covers the interactions of submonolayer \\ amounts of gold with semiconductor surfaces of these two types and subsequent film \\ growth to a thickness of a few monolayers.
}

Studies of gold films have a long history and the use of evaporated gold films to protect and to gild surfaces emerged more or less in parallel with the development of vacuum techniques. Since then, films of gold have been studied to a greater extent than those of any other metal, because of both the relative simplicity of gold evaporation and the resistance of gold to chemical attack duting and after the deposition process. The literature on gold films therefore constitutes a large proportion of the literature on metal films in general. Although the mechanisms of growth of metal films have been the subject of excellent reviews such as those of Lewis and Anderson (1), Robins (2), and Le Lay and Kern (3), these articles have tended to concentrate on growth from the nucleation stage onwards. Much less attention has been paid to the pre-nucleation stage.

In the present review, attention will therefore be focussed mainly upon this pre-nucleation stage and specifically upon the interaction of evaporated gold with surfaces and the growth of the first few atomic monolayers of films of this metal. Particular attention will be paid to the early stages of growth of gold films on semiconductor surfaces as a large tesearch effort has been concentrated recently in this area. The reason for this is the extensive use of such films in the establishment of electrical contacts of the ohmic and Schottky types.

Gold films on silicon and GaAs usually form Schottky barriers even at monolayer (ML) thicknesses. A detailed knowledge of the mechanisms by which they are formed on these semiconductors is therefore of great importance. The film growth has been found to differ according to whether it occurs on clean ordered surfaces or upon surfaces which are either disordered per se or as a result of the presence of contaminants.

For the study of the growth of gold films especially at monolayer level on clean or ordered surfaces, such as those of clean silicon or GaAs, ultra high vacuum (UHV) techniques and analytical methods such as Auger electron spectroscopy (AES), photoelectron spectroscopy (PES) and electron diffraction have been all-important. Initial film growth is characterized by strong interactions between gold atoms and the substrate surface, leading to intermixing of the metal and the substrate. At low temperatures, typically room temperature, the first monolayer of gold is deposited in a disordered state, and gives rise to the subsequent formation of a mixed compound layer (silicide or alloy) upon which nucleation of gold may occur. At elevated temperatures (typically several hundred Celsius), reconstructions occur within the first monolayer of gold on silicon. Interdiffusion is fast and in thicker films of gold, on GaAs for example, leads to a thick intermixed region. The presence of small controlled amounts of impurities on clean surfaces of silicon and GaAs leads to a marked change in the surface reactions with evaporated gold. With increased impurity concentration the growth mechanisms gradually take on the characteristics of film growth on disordered surfaces. Thus, growth of gold films upon contaminated or disordered surfaces can be described in terms of weak reactions between the metal and the substrate, leading to early nucleation. The classical studies of these processes were carried out using transmission electron microscopy (TEM).

\section{Factors Influencing Metal Film Growth}

The properties of evaporated metal film contacts on semiconductor surfaces depend on the interface between the metal and the semiconductor Important physical characteristics of the interface are the geometrical and electrical ones, particularly the degree of epitaxy and the density of interfacial states. Growth of epitaxial metal films depends in the first instance on the degree of misfit between the lattices of the substrate and the film. A certain misfit is accommodated by an interfacial strain which depends not only on the lattice misfit but also on film thickness and the geometry of interfacial dislocations $(4,5)$. The interface between the substrate and the film represents a discontinuity in the crystallographic periodicity and consequently gives a characteristic electronic structure. For example, when gold is deposited on GaAs (011), electronic interface states (not present on the clean surface) are formed at the interface. Such states are induced by the metal atoms and are important for the features of the Schottky barrier 

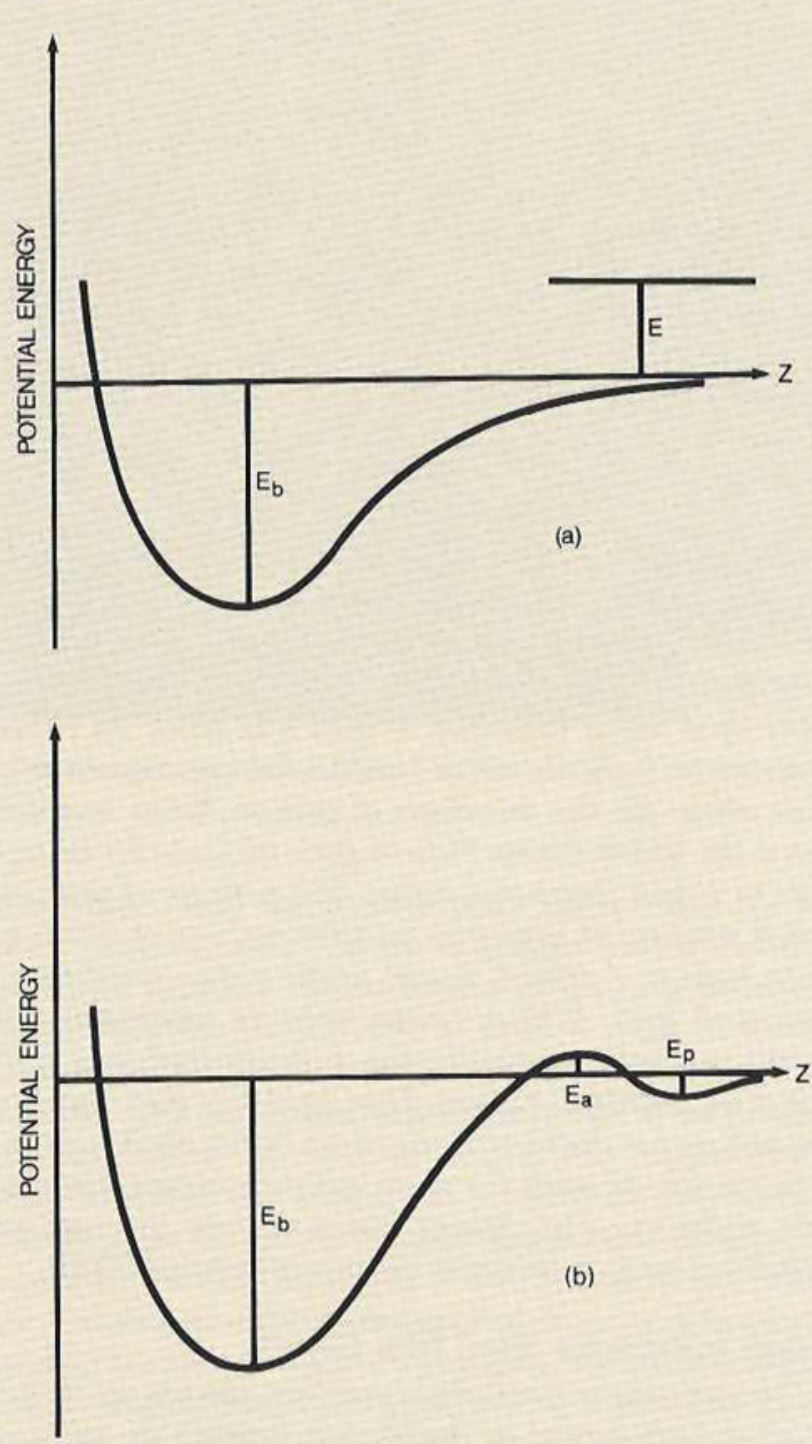

Fig. 1 The principal potential energy diagrams for adsorption. Diagram (a) represents an atom with kinetic energy $\mathrm{E}$ arriving at a site with binding energy $E_{b}$. Diagram (b) shows a weak binding energy $E_{p}$ typically that for physisorption in a so called precursor state which might precede the stronger binding represented by $E_{b}$. Note the activation energy $E_{a}$ necessary to give the atom stronger binding

The reactions at the interface viz. between the metal atoms and the substrate surface atoms have attracted increased interest during recent years. For their study it is natural to use a single crystal substrate having a well defined, clean and ordered surface. Using electron spectroscopy and electron diffraction techniques the surface composition and structure can be studied as a function of deposition when an interface forms. During deposi- tion, the arrival of single metal atoms at the surface leads to a number of events including refraction and inelastic and elastic reflection. However the most important of these surface events in film growth are those leading to the retention of metal atoms, namely physisorption (physical adsorption) and chemisorption.

Detailed knowledge of these phenomena for metal atoms is not as well developed as for gas atoms. Usually, metal atoms are rather weakly bonded to the substrate surfaces and this leads to surface diffusion. The weak bond between adatoms and substrate atoms is characteristic of physisorption. Since this concept is central to initial film growth, a short discussion of it is warranted

In physisorption, no charge transfer takes place but the attractive force is provided by the dipole moment created by mutual polarization of the adatom and the neighbouring surface atoms (Van der Waals forces). The potential diagram representing physisorption is depicted in Figure 1. An atom with energy $E$ (kinetic energy and vibrational energy, which are determined by the temperature of the atom) arrives at the surface, loses the energy $E+E_{b}$ (binding energy) and attaches to the surface by exciting phonons. The bonding energy of a physisorbed atom is typically 0.2 to $0.4 \mathrm{eV}$. After the adsorption stage we can distinguish between the different surface events described below which give rise to various growth modes:

- Chemical reaction

- Alloying

- Interdiffusion

- Surface growth

In chemisorption there is an exchange of electrons between the adatom and the substrate, which results in the formation of a chemical bond providing a binding energy higher than that afforded by physisorption. In the alloying process a surface alloy is formed in which substrate atoms are substituted by adatoms and the crystallographic structure is preserved in regions of microscopic dimensions. Interdiffusion can be understood as a diffusion of adatoms into the substrate and substrate atoms into the growing film. It results, typically, in substrate material being found on the outer surface of the film. The intermixed region, that is the interface, can be thick, of the order of tens of nanometres. The surface growth of films is dependent on a number of factors, including the degree of the substrate surface ordering and deposition temperature.

\section{Growth Modes of Metal Films}

Three different models of initial film growth, are shown in Figure 2:

(1) Layer or epitaxial growth (Frank-Van der Merwe mode) in which film growth is related to the crystallographic orientation of the substrate surface (for example, gold on gold) 


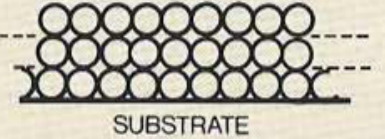

(a)

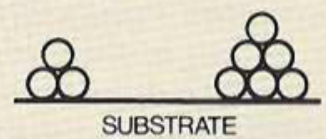

(b)

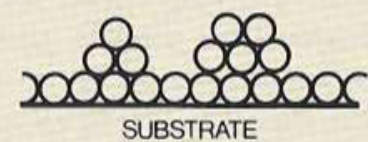

(c)

Fig. 2 The Frank-Van der Merwe (a), Volmer-Weber (b) and StranskiKrastanov (c) modes of growth of metal films. These growth modes, especially Volmer-Weber, are discussed extensively in (1)

(2) Island growth (Volmer-Weber mode) which is characterized by a weak bonding to the substrate compared to the bonding between the adatoms (for example, gold on alkali halides)

(3) Intermediate growth (Stranski-Krastanov mode) which starts with the formation of a monolayer or a submonolayer, but on top of this there is nucleation, leading to island growth (for example, gold on silicon oxide).

Other types of growth processes involve chemical reactions, such as oxide formation when aluminium grows on an oxide substrate; alloy formation, when gold grows on silver; and compound formation, for instance when gold is deposited on clean semiconductor surfaces.

\section{Nucleation}

For many years $(1,2)$ the conventional technique for studying initial film growth was TEM. Using this, early film growth can be detected only after the adatoms have formed small metal nuclei with sizes exceeding the resolving power of the technique. Consequently when TEM can detect the film growth several elementary surface processes, such as adsorption, surface diffusion, chemical reaction or nucleation have already occurred. Therefore, TEM is of limited use when studying the prenucleation growth characterized by interaction between adatoms and surface atoms. At the onset of nucleation, growth is dominated by the adatom-adatom interaction. The surface processes become more complex as adatoms and small particles diffuse on the surface and rapidly form larger particles (2). Experimental evidence (6) for such a migration of sub-microscopic gold particles has been obtained by TEM. A minute amount of material was deposited onto $\mathrm{NaCl}$. After annealing at room temperature nucleation occurred and growth of detectable particles was seen when using high resolution TEM indicating a considerable surface mobility and coalescence even at room temperature.

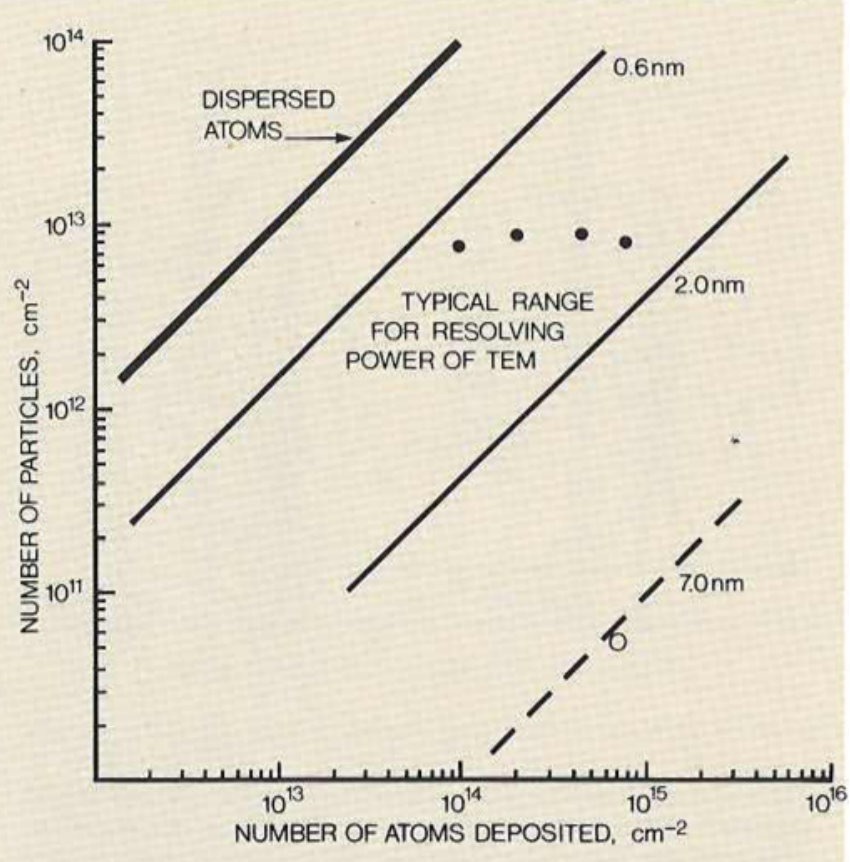

Fig. 3 A schematic representation of the number of metal particles formed as a function of the number of metal atoms deposited on a substrate. The lines represent different average thicknesses of films having equally sized spherical particles. A few experimental points, from TEM studies, are shown for gold on carbon. After $(39,40)$. It is evident that a large number of atoms has to be deposited before particles are observed in TEM

The increase in surface diffusion rate with temperature increases the nucleation tate. In Figure 3, the relationship between the density $n$ of nucleated metal particles on the surface and the density $N$ of atoms deposited onto the surface is illustrated. For a fcc-crystal with lattice constant $a$ this relationship is

$$
N=n \frac{16}{3}\left(\frac{r}{a}\right)^{3}
$$

where $r$ is the radius of a circular particle.

The typical resolution of TEM is in the range 0.5 to $1.0 \mathrm{~nm}$. As shown in. Figure 3, gold particles with such sizes have been found in very thin films. At elevated substrate temperatures both adatoms and nuclei diffuse over the surface resulting in coalescence and rapid growth of larger particles. The effect of enhanced surface mobility is illustrated in Figure 3 by the line $r=7.0 \mathrm{~nm}$.

To investigate the pre-nucleation stage very small amounts of gold, typically below $1 \mathrm{ML}$, must be deposited onto the surface and methods other than TEM must be used. Since the development of UHV techniques, several methods have been used for the controlled detection of metal atoms on a surface and for the determination of their chemical states. Methods such as AES and 


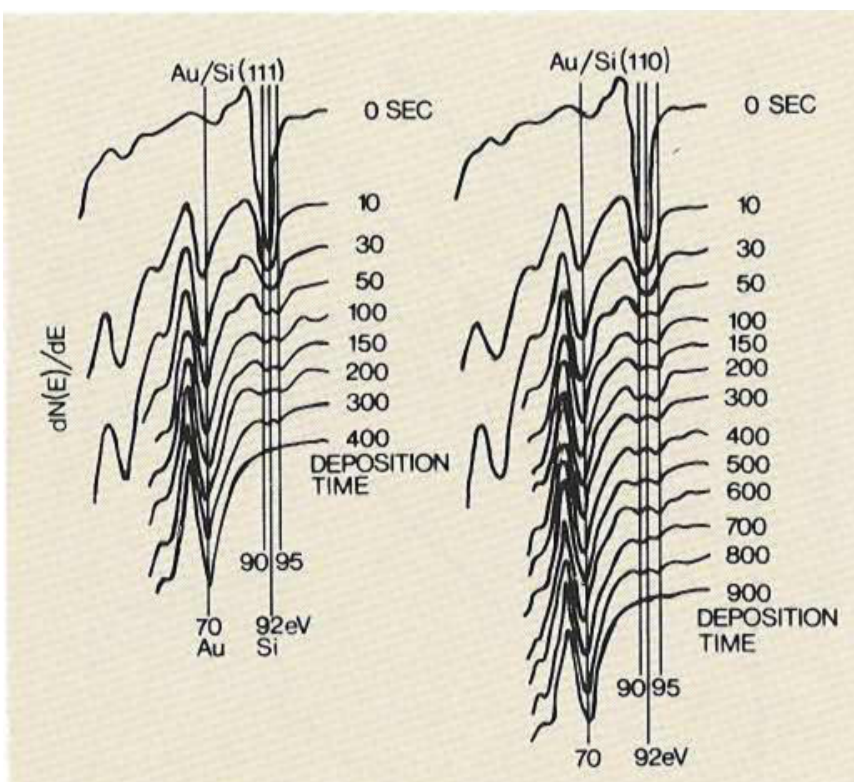

Fig. 4 Auger spectra corresponding to increasing thicknesses of gold deposited on clean silicon surfaces. It should be noted that the silicon peak splits and decreases in magnitude when surface coverage by gold increases

PES rely on electronic excitations of the atoms. AES and PES (synchrotron tadiation) have a detection limit of $10^{-4}$ to $10^{-3}$ ML. These two important methods are described in several textbooks $(7,8)$ where their usefulness for investigating initial film growth is also explained.

\section{Growth of Gold on Silicon}

AES has been widely used for investigating gold films on silicon, and through the observed splitting of a silicon Auger peak has provided evidence for silicide or alloy formation at the surface. Available data indicate that there is a structural transformation at about $400^{\circ} \mathrm{C}$, above which films usually exist as single crystals. Furthermore, it seems that there is a critical coverage of about 1 ML. Below this the gold atoms stay on top of the surface, while above $1 \mathrm{ML}$ there is intermixing or nucleation, depending on the temperature.

\section{Gold on Si(111) at Room Temperature}

TEM was used for the first studies of the growth of thin gold films on surfaces of $\operatorname{Si}(111)$ (9). Micrographs of films with thicknesses of 1.0 to $3.0 \mathrm{~nm}$ revealed a mixing of the initially deposited gold (equivalent thickness $=1.0 \mathrm{~nm}$ ) and the silicon. Above this thickness gold particles nucleated to give the Stranski-Krastanov growth mode. The observed mixing of gold and silicon at the interface is in agreement with earlier work from which it was concluded that gold atoms diffused through single crystals of silicon (10). The reverse situation - diffusion of silicon atoms through gold films - was observed by Hiraki et al. (11 to 14). In their work 20 to $400 \mathrm{~nm}$ thick gold films, deposited on the surface of thick silicon substrates, developed a silicon oxide at the vacuum interface when it was heated in an oxidizing atmosphere. Apparently the interaction at the gold/silicon interface provided silicon atoms which diffused through the gold layer. This naturally generated increased interest in the formation of the gold/silicon interface.

In one experiment the $\mathrm{Si}\left(\mathrm{L}_{2,3} \mathrm{VV}\right) 92 \mathrm{eV}$ AES-peak (referred to as LVV in the following) was studied during slow evaporation of gold onto Si(111). The deposition of gold resulted in a thin surface layer characterized by splitting of the LVV-transition into two peaks at 90 and $95 \mathrm{eV}$ respectively (15), as is to be seen in Figure 4. Later (16), it was revealed that the double peak started to develop at $0.5 \mathrm{ML}$ surface coverage and dominated the LVVtransition at 2.5 ML. Comparing the double-peaked AES spectra with the spectra from an amorphous gold-silicon alloy quenched from the liquid state, which also exhibited a chemical shift, it was concluded that the splitting of the LVV peak following deposition of gold onto the silicon surface was a result of the formation of a gold-rich ( 70 per cent) amorphous surface alloy (17). Heating such an amorphous gold-silicon mixture resulted in a structural transformation to one of several crystalline phases. These results stimulated studies of the thermal behaviour of the surface alloys formed by gold and silicon.

Green and Bauer (18) investigated by low energy electron diffraction (LEED) and AES the structure of the gold silicides, resulting from the deposition of 10 to $100 \mathrm{~nm}$ of gold. Again, it was found that the room temperature deposit was amorphous as it gave no LEED pattern. Furthermore, the interaction at the gold/silicon interface (diffusing silicon atoms) produced a silicide on both sides of the gold film, in agreement with carlier results (1). It was also shown that annealing the silicide at temperatures between 200 and $375^{\circ} \mathrm{C}$ produced gold-tich crystalline silicide layers, in agreement with observations made during the annealing of quench-condensed amorphous goldsilicon mixtures (17). The silicide layers had a maximum thickness of $3.0 \mathrm{~nm}$, and were separated by a thicker gold layer containing less silicon. The structure of the silicide material could not be determined by measurements of relative Auger peak magnitudes, but Green and Bauer suggested for it the composition $\mathrm{Au}_{5} \mathrm{Si}$.

In the latter studies the gold film thicknesses were $10 \mathrm{~nm}$ or more and the interface between the semiconductor and the metal consisted of a mixed region. However, the split AES spectra, which were evidence for this, were insensitive to the composition of this mixed region. Thus, it seems that an alloy could be formed as well as the silicide. To obtain information about the formation of such an alloy one must study the condensation of the first metal atoms onto the silicon surface, that is the 


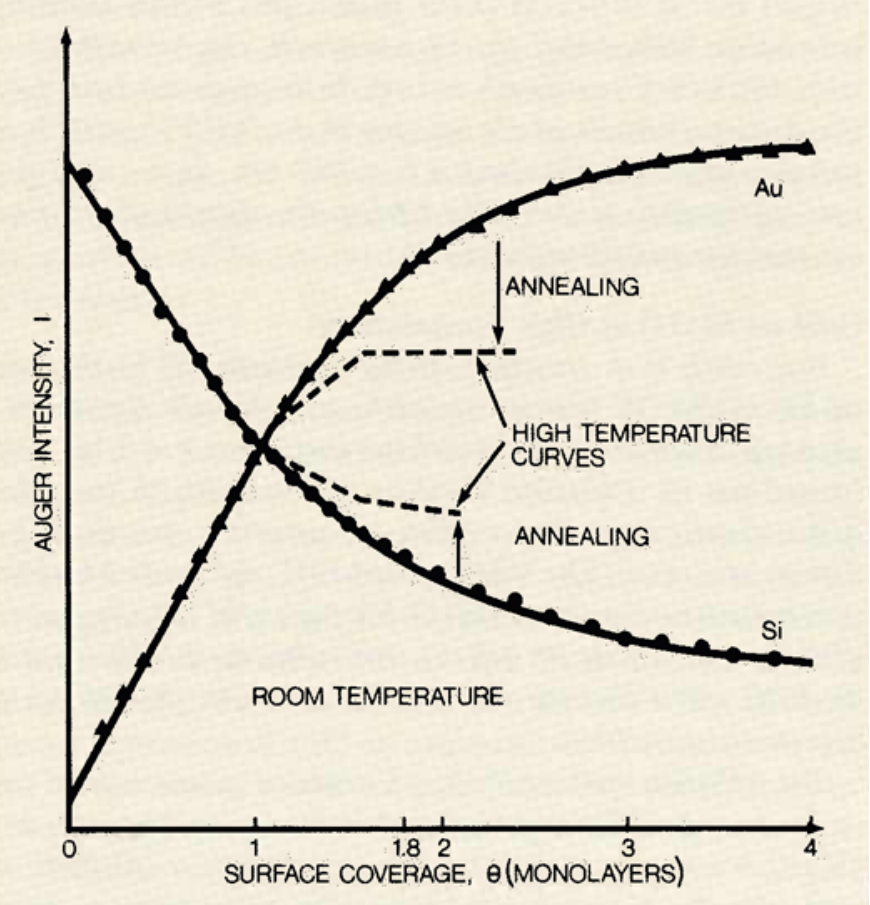

Fig. 5 Auger intensities from gold and silicon as a function of surface coverage $\theta$ by gold. See also Figure 7

growth of metal monolayers on ordered silicon surfaces.

AES, having a sensitivity of $10^{-3} \mathrm{ML}$, gives a unique possibility of following the growth of metal monolayers. Successive measurements during deposition were made by Le Lay and Faurie (19). The LEED pattern exhibited a gradual disappearance of the $\mathrm{Si}(111)-7 \times 7$ surface reconstruction with deposition of gold. As shown in Figure 5, simultaneous AES measurements revealed a linear increase and decrease of the $\mathrm{Au}\left(\mathrm{N}_{6,7} \mathrm{VV}\right)$ and $\mathrm{Si}(\mathrm{LVV})$ signals respectively. Thus, the first monolayer of gold atoms adsorbed on a Si(111)-7x7 surface as a disordered structure, but without intermixing. The previously discussed surface alloy or silicide, as determined from the splitting of the Si (LVV) transition, developed only at $4 \mathrm{ML}$. Annealing of a room temperature deposit caused the amorphous surface structure to crystallize. This is discussed below.

Studies of the splitting of the Si (LVV) Auger transition are very important as they indicate a chemical change. Such shifts of the electronic binding energy levels give supplementary information about the electronic structure of the interface region. In an investigation by Braicovich et al. (20), the intensity of core level excitations, relative to the intensity of Auger transitions, and the energy distribution curves (EDC) from the valence band, were studied by PES during the deposition of gold onto Si(111). The intensity is a measure of the surface concentration while the

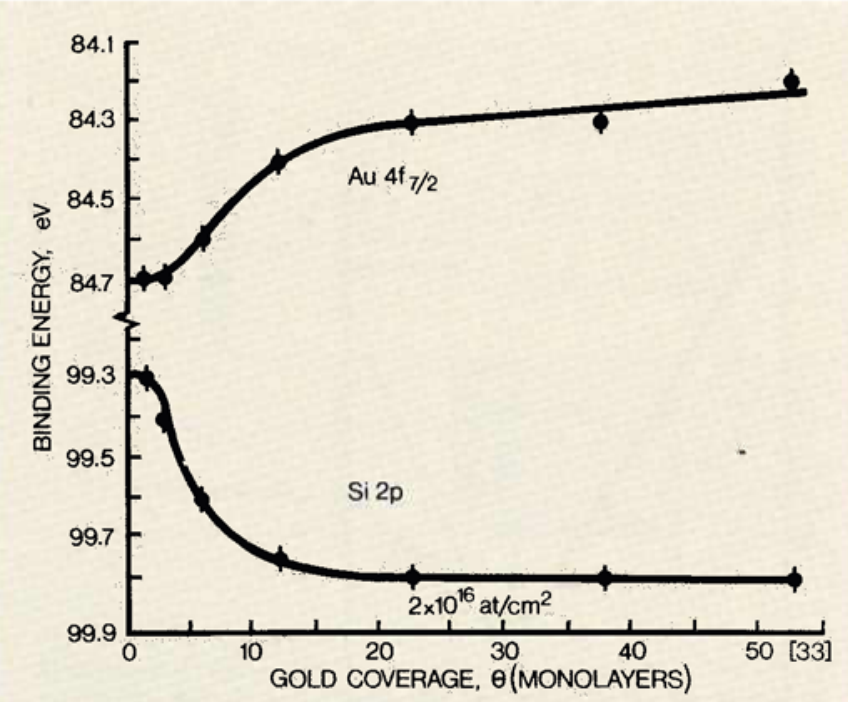

Fig. 6 The binding energies, referred to the Fermi level, of the $\mathrm{Au} 4 \mathrm{f}$ and $\mathrm{Si} 2 \mathrm{p}$ electron levels as a function of coverage by gold. The latter is expressed in monolayers referred to the atomic site density on the silicon surface (and in parentheses for a gold surface). The absolute density of gold atoms is also given. It is evident that the gold level changes towards the binding energy level for bulk gold while the silicon level changes from the value for bulk silicon

energy shift gives evidence of atomic interactions. Changes of the EDC indicate transitions from states of dispersed atoms to nucleated particles. The intensities of the core level binding energies from $\mathrm{Au} 4 \mathrm{f}_{7 / 2}(84.0 \mathrm{eV})$ and $\mathrm{Si} 2 \mathrm{p}(99.3 \mathrm{eV})$ were measured as a function of the coverage, $\theta$, of gold. (The indicated energies are for bulk materials). The binding energies for these levels changed with the coverage as shown in Figure 6. It is evident that the growth could be divided into three stages corresponding to three different physical situations. At low coverages, $\theta \leq 2 \mathrm{ML}$, the most dramatic change was the rapidly reduced emission from the surface states indicating a disturbance of the surface by the deposited gold atoms. Furthermore, EDC of the valence band emission indicated that the gold was atomically dispersed in the surface region in which it underwent reaction with the silicon. At $2 \mathrm{ML}$ coverage the intermixed region extended to a depth of approximately $1.5 \mathrm{~nm}$, with 70 per cent of the gold in the uppermost $0.5 \mathrm{~nm}$ surface region.

Deposits with thicknesses in the range $2 \leq \theta \leq 15 \mathrm{ML}$ exhibited a gold emission which gradually became more bulk-like. The $A u$ ff binding energy level shifted towards the value for bulk gold while that of Si $2 \mathrm{p}$ shifted in the opposite direction. This points to the formation of a gold-rich phase in which the bonding of silicon is quite different from that existing in a silicon crystal. Only a slow increase in the gold content was measured over the third range of film thickness with $\theta>15 \mathrm{ML}$ 


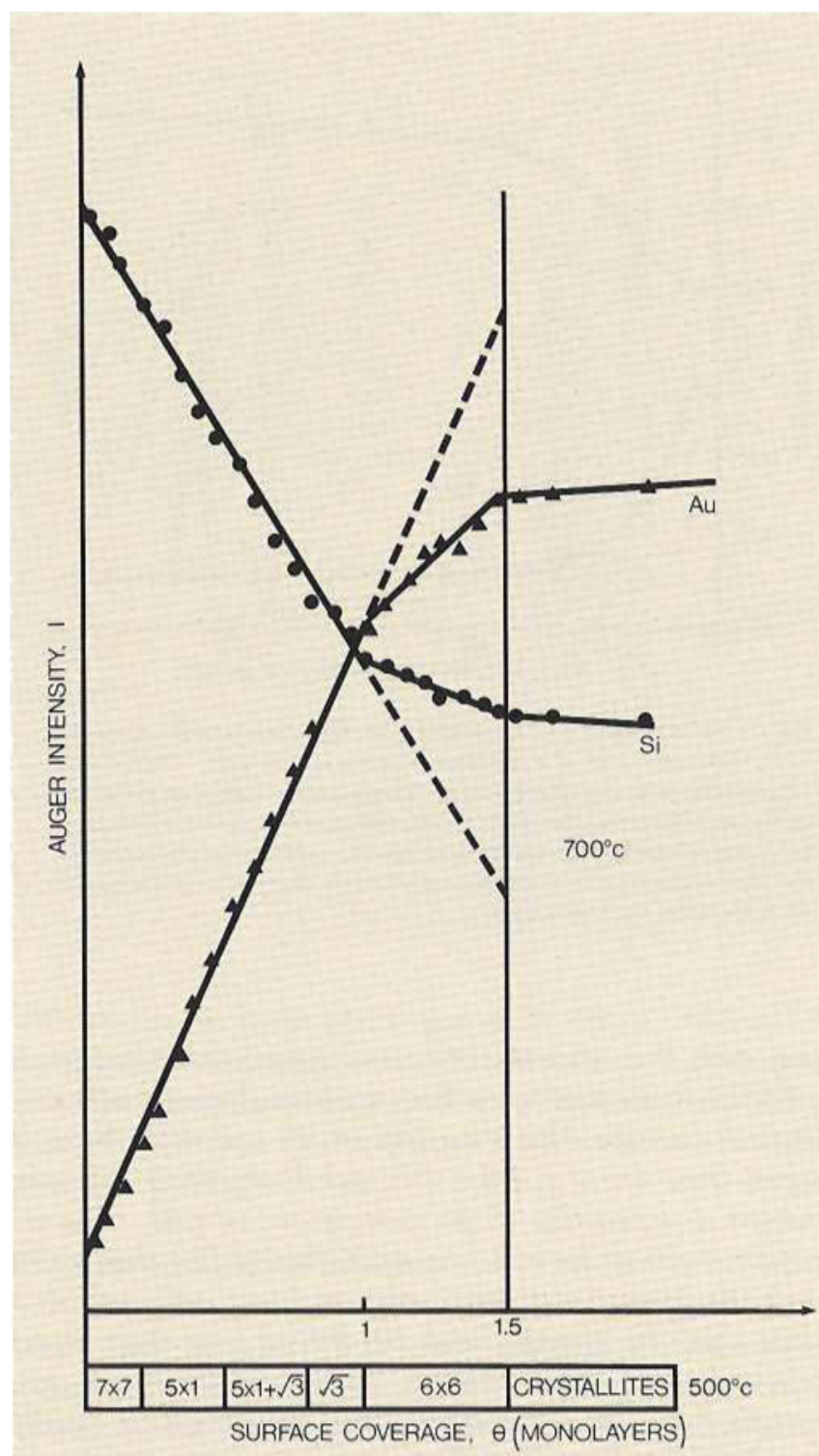

Fig. 7 Auger intensity curves for gold and silicon as a function of gold coverage when deposition occurs at $700^{\circ} \mathrm{C}$. The surface domains as detected by LEED are indicated below the figure

(see Figure 6). There was evidence, however, of segregation of a fraction of a monolayer of dispersed silicon atoms to the topmost surface gold layer.

\section{Gold on Si(100) at Room Temperature}

Deposition of gold on $\mathrm{Si}(100)$ was studied by Oura et al. $(21,22)$. Films with thicknesses ranging from 0.6 to $30 \mathrm{~nm}$ were deposited at close to room temperature and studied by AES and LEED after various heat treatments. After deposition of $1.0 \mathrm{~nm}$ of gold the Si(100)-(2x1) LEED pattern had degraded leaving only a high background. At $3.0 \mathrm{~nm}$ a weak ring became detectable which was considered to have been produced by a gold silicide layer, because of the splitting of the $\mathrm{Si}$ (LVV) peak. Further deposition of gold, from 6.0 to $30.0 \mathrm{~nm}$, gave a spot pattern interpreted as $\mathrm{Au}(111)$, having six azimuthally rotated orientations, parallel with $\mathrm{Si}(100)$.

\section{Gold on Si(111) at High Temperatures}

Braicovitch et al. annealed a sample consisting of $55 \mathrm{ML}$ gold on $\mathrm{Si}(111)$ for 10 minutes at $550^{\circ} \mathrm{C}$ and showed that a new, silicon-rich, layer characteristic of low coverages ( $\theta \leq 1 \mathrm{ML}$ ) was formed but no nucleation could be detected (20). A more detailed investigation of the effect of annealing was made by Abbati et al. (23). The valence band EDC was studied by PES after various heat treatments of $10 \mathrm{ML}$ thick gold films deposited at room temperature on $\mathrm{Si}(111)$. A continuous modification of the EDC and a decrease of the gold signal took place at up to approximately $400^{\circ} \mathrm{C}$.

The diffusion coefficient of gold in silicon cannot explain the rapid decrease of the gold signal with rise in temperature. Therefore it was concluded that the intermixing, or diffusion, of gold into the silicon was a chemically driven process. Heat treatments above $400^{\circ} \mathrm{C}$ did not change the EDC. No evidence was found for the formation of a stable compound. However, since the gold silicon eutectic temperature $\left(370^{\circ} \mathrm{C}\right)$ is close to $400^{\circ} \mathrm{C}$ there is, in fact, a possibility that a eutectic compound was formed.

When condensation was at temperatures above $400^{\circ} \mathrm{C}$, the film growth followed the Stranski-Krastanov mode, that is one monolayer was built up upon which nucleation took place (24). This first monolayer exhibits several surface reconstructions. The complexity of this surface ordering is illustrated in Figure 7 where the different surface domains are shown. These data and isothermal desorption experiments showed that $2 \mathrm{D}$ ordered phases were formed before $3 \mathrm{D}$ phases started to grow at $\theta=1.5$ ML. The data enable the construction of a $2 \mathrm{D}$ phase diagram showing the relationship between the substrate temperature and surface coverage (24).

\section{Gold on Si(100) at High Temperatures}

Heat treatment of gold films of thicknesses 0.6 to $30 \mathrm{~nm}$ on surfaces of $\mathrm{Si}(100)$ produced superstructures (22). The rather complex relationships between temperature, film thickness and surface structure are illustrated in Figure 8. Here it is obvious that $400^{\circ} \mathrm{C}$ divides the temperature range into two regions, with the crystalline gold silicide or alloy being stable only in the low temperature region. Annealing to the high temperature region leads to agglomeration of the film and thermal desorption of both the gold-rich particles and the intermediate $2 \mathrm{D}$ regions with gold-induced superstructures. 


\section{Gold on III-V Compounds}

In this section the interaction of gold with surfaces of III-V compounds, particularly GaAs, is discussed. The industrial application of gold layers to semiconductors in the fabrication of Schottky contacts $(25,26)$ is usually carried out using materials with contaminated surfaces. Therefore the growth of gold on such surfaces will be discussed before its growth on clean surfaces is reviewed.

\section{Growth on Contaminated GaAs Surfaces}

The type of surface contamination present on GaAs is dependent on the chemical treatment to which the material has been subjected. Typical contaminants are oxides $\left(\mathrm{Ga}_{2} \mathrm{O}_{3}\right.$ and $\left.\mathrm{As}_{2} \mathrm{O}_{3}\right)$ and carbon. In the presence of $\mathrm{Ga}_{2} \mathrm{O}_{3}$ and $\mathrm{As}_{2} \mathrm{O}_{3}$, deposition of 'reactive' metals (for example titanium, chromium, aluminium or magnesium) results in chemical reaction and the formation of a layer of a metal oxide between the GaAs substrate and the metal film (27). Such chemical reaction does not occur, however, during deposition of gold on oxide-contaminated surfaces, and the mechanism of growth of gold films may therefore be expected to differ from that of reactive metal films.

The influence of carbon contamination on the growth of gold films on GaAs was investigated by Vermaak, Snyman and Auret(28). Substrates with three different surface concentrations of carbon were produced (Table I). Thereafter, discontinuous gold films with thicknesses of $5.0 \mathrm{~nm}$ were grown on the substrates at room temperature. It was found that a carbon concentration of 36 per cent completely destroyed the epitaxial influence of the substrate, the gold films being polycrystalline. A carbon concentration of 25 per cent gave a preferred orientation of the islands in local areas, while 11 per cent carbon led to epitaxial films with local areas of polycrystalline morphology. The latter was also observed at carbon concentrations as low as 3 per cent. Gold grown on a clean GaAs(001) surface showed perfect epitaxial growth with $(100)_{\mathrm{Au}} / /(100)_{\mathrm{GaAs}}$ and $\left.<100\rangle_{\mathrm{Au}} / /<100\right\rangle_{\mathrm{GaAs}}$. This corresponds to a lattice misfit of approximately 32 per cent.

The growth of gold at 150 to $350^{\circ} \mathrm{C}$ on $\mathrm{GaAs}(110)$ surfaces contaminated with both oxide and carbon gave epitaxial $\mathrm{Au}(110)$ films despite the initial $1.0 \mathrm{~nm}$ of gold exhibiting a disordered structure (29). At low temperatures, below $150^{\circ} \mathrm{C}$, the initial degree of disorder was even greater and epitaxial ordering was only observed at a thickness of $30 \mathrm{~nm}$.

It is evident that it is possible to grow epitaxial gold films on GaAs even in the presence of limited contamination, typically less than 1 ML. A characteristic of the initial gold growth, as observed by electron diffraction, is the formation of a disordered structure before the epitaxial structure is formed.

\section{Growth on Clean GaAs(110)}

The creation of intial surface disorder on a clean ordered sur-

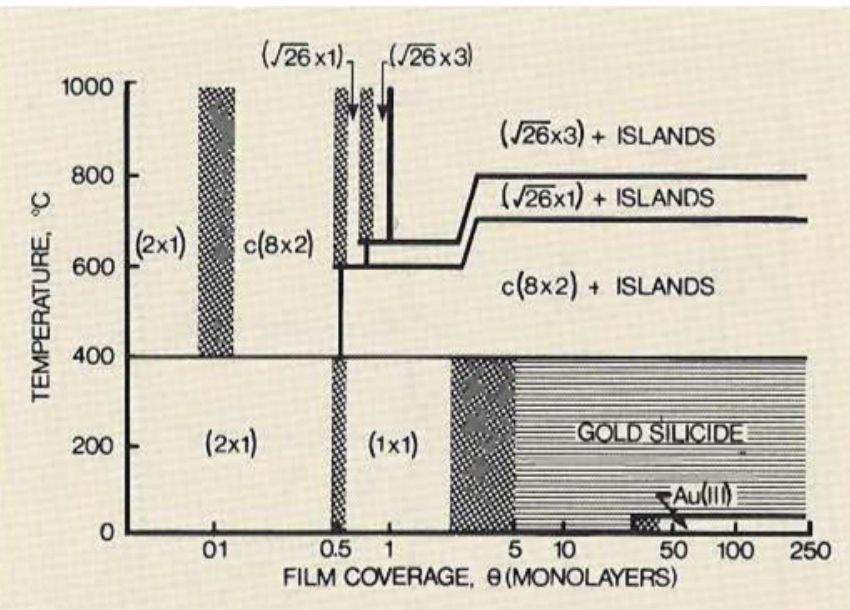

Fig. 8 Schematic illustration of the relationship between substrate temperature, surface structure and surface coverage by gold for the system $\mathrm{Au} / \mathrm{Si}(100)$. Island growth starts above $1 \mathrm{ML}$ coverage for deposition temperatures above $400^{\circ} \mathrm{C}$. At room temperature, an oriented overgrowth, $\mathrm{Au}(111)$, is obtained for thick gold layers, such that the orientation effect propagates through the thin layer shown as a silicide in the figure

face of $\mathrm{GaAs}(110)$ has been observed not only by electron diffraction but also by PES. Thus deposition of 1 to $3 \mathrm{ML}$ of gold removed intrinsic electronic surface states indicating severe structural changes of the surface (30).

In an extensive investigation by Chye et al. (31) the formation of gold films on clean and ordered surfaces of III- $V$ compounds was studied by PES at submonolayer and thicker films. The deposition of gold on (110)-oriented surfaces of GaAs, GaSb and InP caused the semiconductor surface to decompose and substrate elements were found on top of the gold layer. This was especially pronounced on $\mathrm{GaSb}$. Here the amount of antimony was constant while the gallium decreased to zeto when the sur-

Table 1

Atomic Per Cent Concentrations of Carbon, Oxygen and Potassium on Chemically Cleaned GaAs(001) Substrates. After (28).

\begin{tabular}{lcccc}
\hline \multirow{2}{*}{ Condition of sample } & \multicolumn{5}{c}{ Sample Number } \\
\cline { 2 - 5 } & & 1 & 2 & 3 \\
\hline Room & C & $33-42$ & $11-12$ & 0 \\
Temperature & O & $4-8$ & $8-10$ & $10-15$ \\
After Annealing & K & - & - & $4-6$ \\
at $500^{\circ} \mathrm{C}$ & $\mathrm{C}$ & $25-36$ & $3-5$ & 0 \\
for 1 hour & $\mathrm{O}$ & 0 & 0 & 0 \\
& $\mathrm{~K}$ & - & - & 0
\end{tabular}



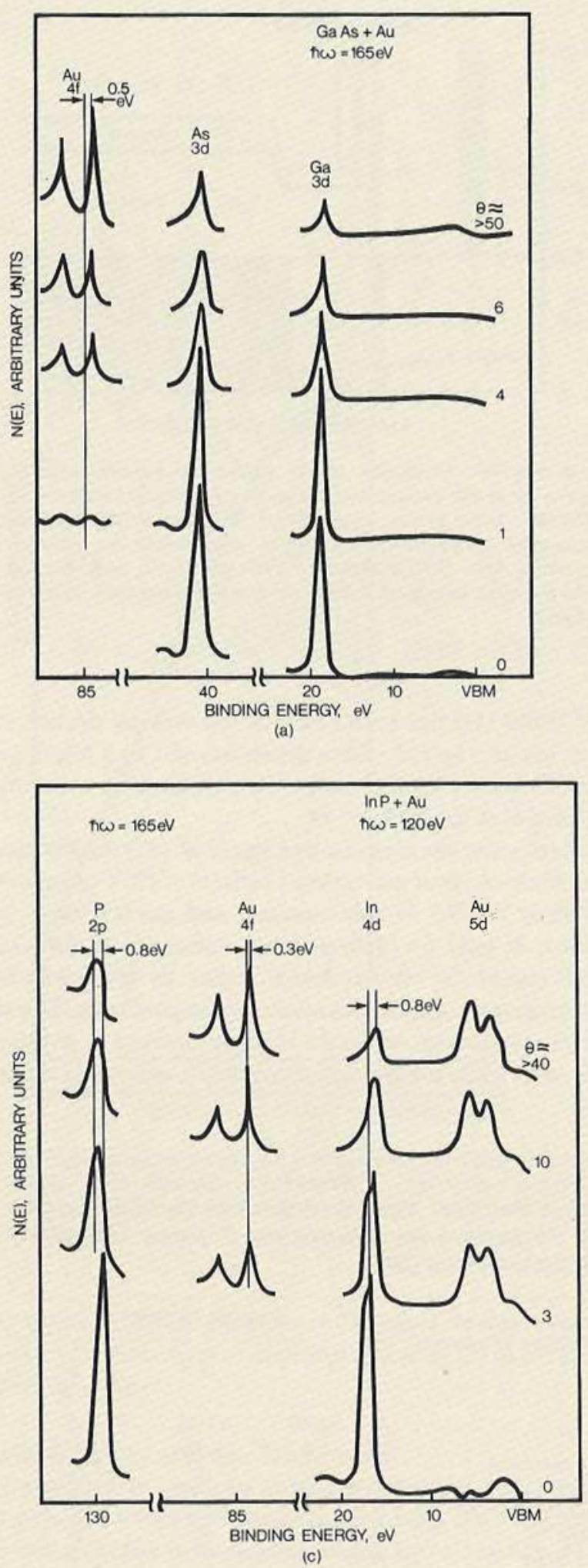

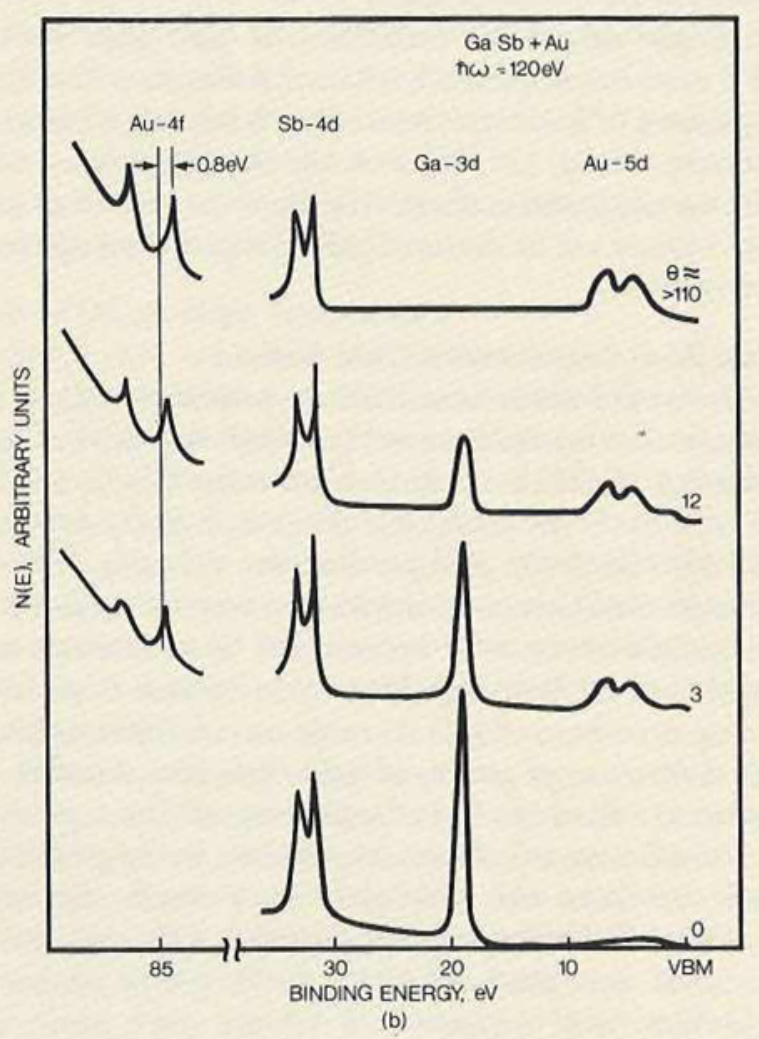

Fig. 9 PES spectra taken with such photon energies that give maximum surface sensitivity. The figures show the various surface coverages achieved when gold is deposited onto (a) GaAs, (b) GaSb and (c) InP. The elements constituting the semiconductor are found on top of the gold film for most coverages

face coverage of gold was increased to $110 \mathrm{ML}$. Similar but less pronounced behaviour was observed for gold on GaAs and InP (see Figure 9). Measurements of the electron distribution curves, using ultraviolet photoelectron spectroscopy (UPS), revealed that the gold was dispersed atomically at the surface for up to 4 to $5 \mathrm{ML}$ (31). The dispersed gold atoms caused disorder in the semiconductor surface at coverage levels as low as $\theta=0.5 \mathrm{ML}$. Thus we can conclude that a surface layer consisting of a mixture of the semiconductor elements and gold is formed after the deposition of a few monolayers of gold.

With increasing gold coverage on $\mathrm{GaAs}(110)$ there is an increase in the As/ $\mathrm{Ga}$ ratio. Consequently, there must be a change in the gallium and arsenic concentrations with depth, as well as in the gold distribution after deposition. Sputter etch profiling using AES has confirmed such compositional depth profiles (31). This development is determined by the relative solubilities, the tendencies to form compounds or alloys and the relative diffusion rates of the elements involved. Valence band spectra have shown that there is no bulk gold (that is, gold particles) at the surface until the deposit thickness grows to 4 to $5 \mathrm{ML}$ (31). Thus, 
it is likely that gold-gallium alloys or compounds (for example $\mathrm{AuGa}_{2}$ or $\mathrm{AuGa}$ ) are formed. Due to the enrichment of the surface in arsenic there is also a possibility of the formation of some free gallium which in turn could enhance the nucleation of gold. The formation of free arsenic and gallium leads to the question of whether this is a result of the interaction of single gold atoms with the surface or an effect which becomes important with increasing gold film thickness. Using Molecular Beam Epitaxy (MBE) for substrate surface production, the controlled interaction between gold atoms and the GaAs surface can be studied for different surface geometries and concentrations of arsenic.

\section{Growth on Clean GaAs(100)}

In order to study the growth of monolayers of gold on GaAs in the presence of excess arsenic, gold was deposited onto $\mathrm{GaAs}(001)-\mathrm{c}(4 \times 4)$ (32). GaAs layers were grown by MBE in an arsenic-rich atmosphere to produce a $\mathrm{c}(4 \mathrm{x} 4)$ surface reconstruction. The samples were left overnight to reduce the arsenic pressure in the growth chamber before the gold deposition. Gold was then deposited at a constant substrate temperature of $200^{\circ} \mathrm{C}$. This temperature was high enough to give a reproducible amount of excess arsenic as measured by AES. After each deposition both surface composition and geometry were analyzed by AES and RHEED techniques.

Figure 10 shows the $\mathrm{Au}\left(\mathrm{L}_{6,7} \mathrm{VV}\right)$ Auger peak-to-peak amplitude as a function of the deposition time for two different deposition rates, $2.1 \times 10^{12}$ and $4.5 \times 10^{12}$ atoms $/ \mathrm{cm}^{2}$.s. The increase of the Auger signal is chatacterized by a linear part which represents equal deposited mass despite the two deposition rates. The initial deviation from linearity represents atoms which do not contribute to the Auger signal as a result of an initially low sticking coefficient or of a rapid diffusion of gold atoms to depths greater than the escape depth of the Auger electrons. To enable the possible change of the Auger signals due to intermixing at the surface to be studied, a long delay $(1 \mathrm{~h})$ was introduced between two particular deposition events (marked with arrows in Figure 10). This resulted in three different ranges as depicted in Figure 11 in which arsenic and gallium Auger signals are shown as functions of evaporation time. As expected, the main feature of these Auger signals was their reduction with gold deposition. However, both the arsenic and gallium signals increased during the delays. A related decrease of the gold signal could also be detected (Figure 10). The slope of the linear decrease with time of the arsenic signal diminished between delays. For gallium, on the other hand the slope increased. The gold signal for the two deposition rates decreased abruptly after a short deposition period and then became almost constant. This and other observations are in tune with an initial build up of surface gold by deposition. This increased coverage then ceases as inward diffusion of gold develops.

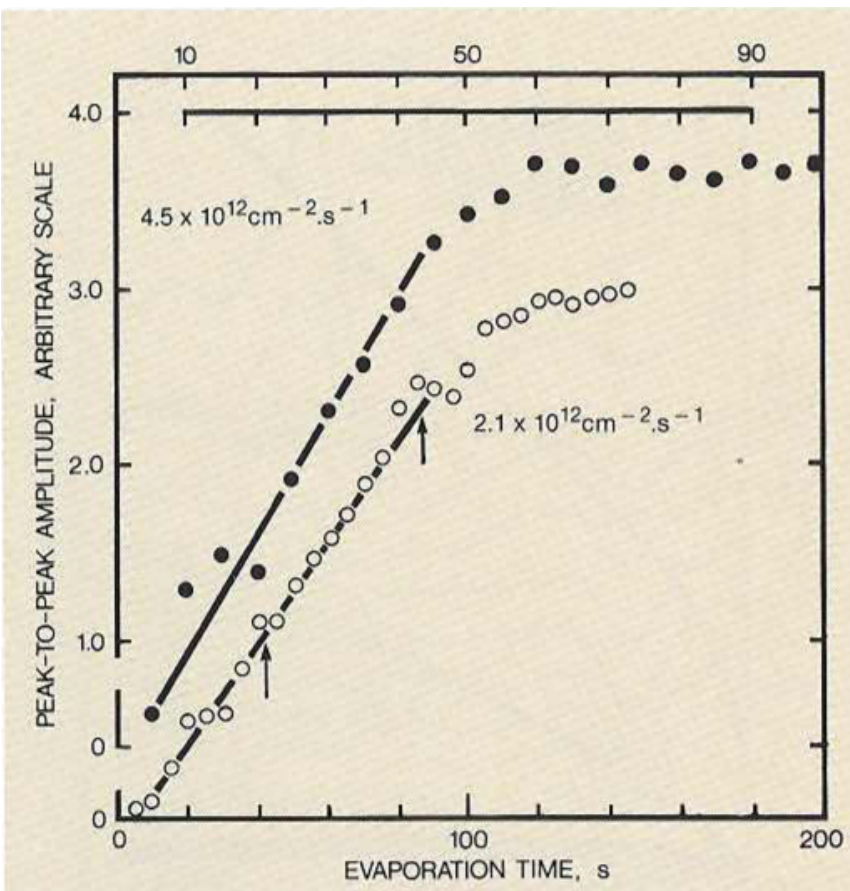

Fig. 10 Variation of the gold Auger signal $(69 \mathrm{eV})$ as a function of evaporation time when depositing gold on GaAs (001)-c(4x4) at room temperature. The figure shows two deposition rates

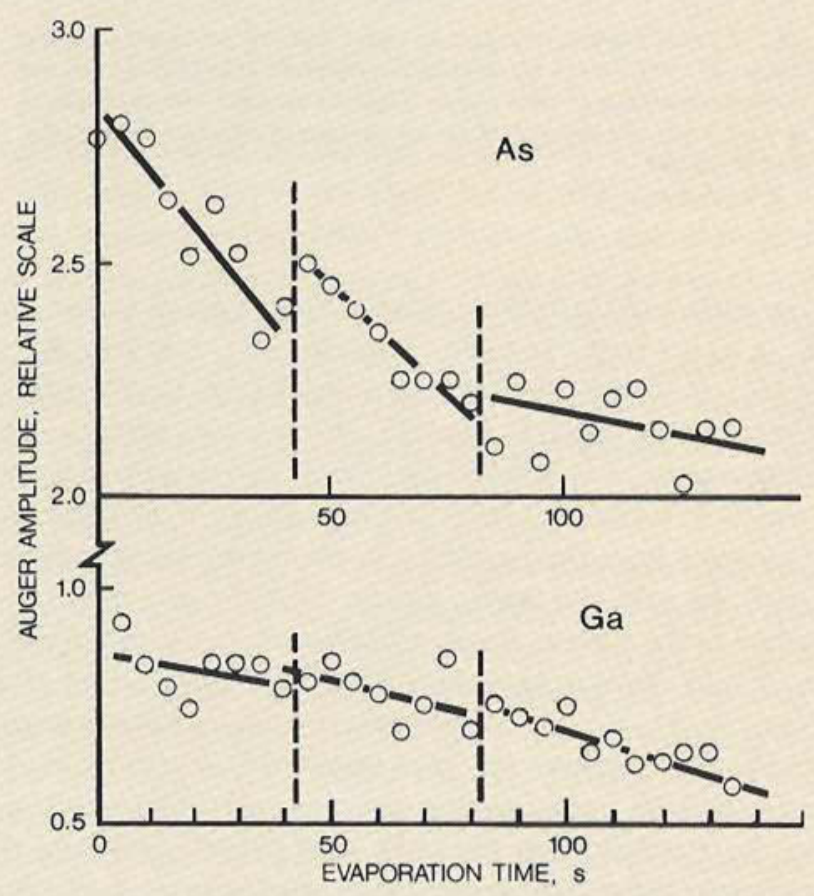

Fig. 11 The arsenic $31 \mathrm{eV}$ and gallium $55 \mathrm{eV}$ Auger signal magnitudes as a function of evaporation time of gold at a rate of $2.1 \times 10^{12} \mathrm{atoms} / \mathrm{cm}^{2} . \mathrm{s}$ 


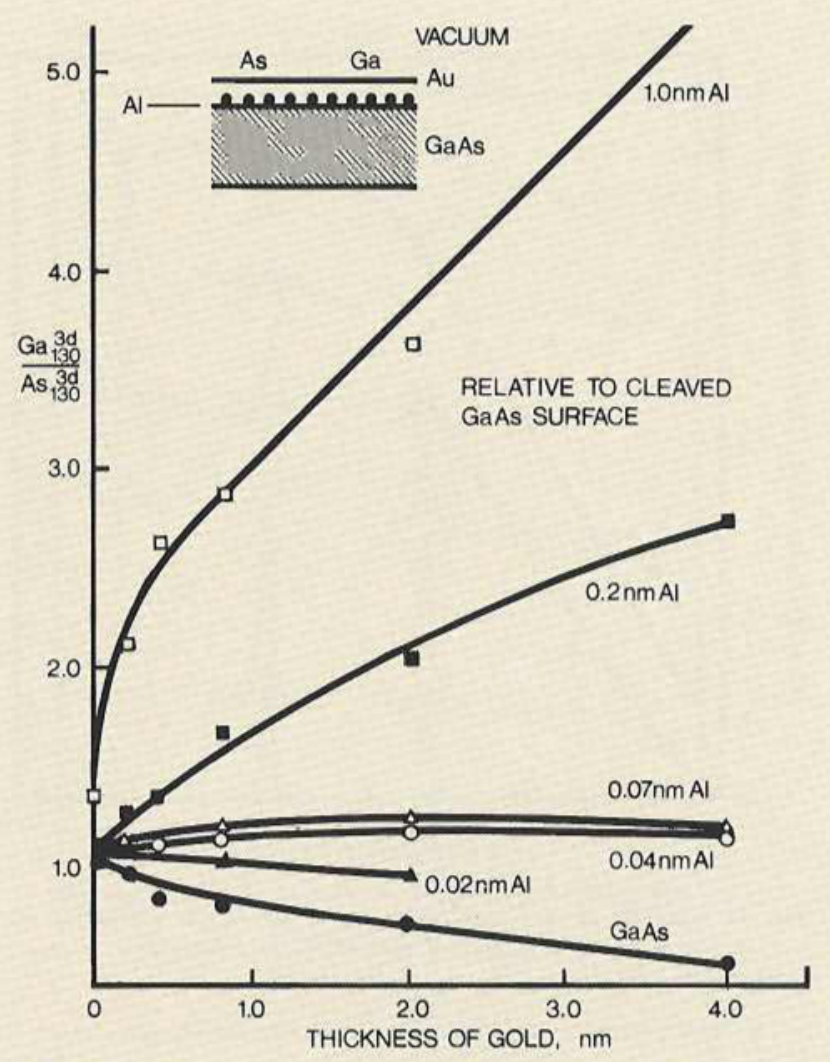

Fig. 12 Ratio between the gallium and arsenic $3 \mathrm{~d}$ peaks (at a photon energy of $130 \mathrm{eV}$ ) as a function of the thickness of gold film with and without aluminium at the interface. The ratio increases with the thickness of gold, while the presence of surface aluminium increases the outdiffusion of gallium

The surface of GaAs (001)-c(4x4) has an excess of arsenic, that is $\theta_{A s}>50$ per cent. At room temperature $\theta_{A s}$ is at least 86 per cent (33). Since the c $(4 x 4)$ surface reconstruction usually exhibits a higher background in RHEED patterns compared to other reconstructions, it is believed that the excess arsenic is dispersed on the (001) surface of GaAs. The intermixing of gold with that surface starts from the beginning of the deposition as indicated by both the gold and arsenic signals. After the gold atoms have attached to the surface, the intermixing occurs via inward diffusion of gold atoms in agreement with observations on the (110) surface $(34,35,36)$. It is interesting to note that deficiencies produced in this way have also been observed (20) at gold/Si(111) interfaces. With increasing deposition of gold on the $\mathrm{c}(4 \times 4)$ surface, the reconstruction gradually disappeared producing the unreconstructed pattern at a coverage of a few per cent of a ML. Thus the unreconstructed surface is observed during the entire linear range extending to $0.3 \mathrm{ML}$, as seen in Figure 10. During further deposition, above $0.4 \mathrm{ML}$, gold and arsenic are assumed to mix mainly in a dispersed state on the surface. The initial rapid decrease of the arsenic signal in Figure 10 (stage I), as compared with the gallium signal, might result from desorption of arsenic from the surface when the gold atoms are deposited on it. The excess energy of the latter is released locally and presumably resulted in desorption of the volatile arsenic.

Without desorption the relative change of the arsenic signal would be expected to be less than that of the gallium signal due to the greater escape depth for arsenic $(0.6 \mathrm{~nm})$ compared with that of gallium $(0.4 \mathrm{~nm})(33)$.

The conclusion that can be drawn about the submonolayer growth at elevated temperatures of gold on $\mathrm{GaAs}(001)-\mathrm{c}(4 \times 4)$ is that intermixing at the surface increases with gold coverage, as well as with substrate temperature. At $300^{\circ} \mathrm{C}$ the intermixing is sufficient to make it difficult to follow the increase of the gold signal in a reproducible way. Three growth stages indicating different rates of intermixing with the substrate, could be detected: (1) Below $\theta=0.3 \mathrm{ML}$ coverage there was a slight interdiffusion, as indicated by the sensitivity of the gold and arsenic signals to deposited mass

(2) In the range $\theta=0.3$ to $0.4 \mathrm{ML}$ there was markedly increased diffusion, as indicated by the reduced coverage dependent AES signal

(3) Above $\theta=0.4 \mathrm{ML}$ coverage the surface interaction was dominated by an intermixing process in which the interdiffusion was so rapid that no increase in the gold signal was observed despite further deposition of the metal.

Comparing the arsenic and gallium AES signals at a gold coverage of $0.5 \mathrm{ML}$ gave evidence for arsenic accumulation at the surface. Furthermore, we can conclude that a submonolayer of gold, in this case about $0.3 \mathrm{ML}$, was built up on the GaAs surface before the intermixing started.

\section{The Influence of Aluminium}

Interdiffusion at the gold/silicon interface is partially inhibited by the presence of oxygen on the silicon surface (20). A similar phenomenon has been observed for the GaAs surface when partially covered by aluminium $(34,35,36)$. Deposition of gold onto aluminium/GaAs did not destroy the bonding between the GaAs substrate and the aluminium atoms, but stimulated diffusion of arsenic and gallium from the substrate to the surface. Thus, gold atoms diffused to below the aluminium layer, and free gallium and arsenic were detected at the surface, that is in the gold film. The amount of aluminium present affected the gold-induced outward diffusion of gallium and arsenic with the result that an increase in the aluminium thickness increased the Ga/As ratio in the gold film (Figure 12). The deposited gold remained dispersed for coverages above 1 ML and no gold particles were formed. Furthermore, evidence was obtained for the development of an enhanced concentration of gallium at the vacuum interface, that is, on top of the gold. 


\section{Growth on Disordered Surfaces}

Disordered surfaces are characterized by a lack of long range order. When examined by RHEED they produce diffuse patterns. Even single crystals may have disordered surface layers if they have been exposed to the ordinary atmosphere and have become contaminated by adsorbed hydrocarbons or other substances. When gold atoms arrive at such surfaces they attach to weak adsorption sites and subsequently undergo surface diffusion. During this process they migrate to sites which have higher binding energies than the average, either as a result of the surface geometry or of the presence of impurities. These conditions provide a high probability of nucleation growth, since the bonding between the gold atoms is higher than that between them and surface atoms. This usually results in weak bonding between the film and the substrate, such as that found between gold films and glass.

In contrast to what is observed on clean single crystal substrates there is usually no relationship betwcen the structure of a substrate with a disordered surface layer and that of a metal film grown upon it. There are, however, exceptions to this $(22,23)$. In this section we shall discuss the formation of gold films on disordered bulk structures and on crystals covered with thick disordered layers, that is, those which produce only diffuse RHEED patterns.

Silicon is very sensitive to exposure to air, and chemisorption of only 0.5 ML of oxygen is enough to generate disordered surfaces on silicon crystals. The presence of these reduces intermixing of the undetlying silicon with deposited gold. Such surfaces therefore tend to promote the formation of gold nuclei (20). Analyses by AES and RHEED have been effective in establishing these facts.

Auger spectra (first derivatives) were recorded for various coverages of gold as shown in Figure 13. On the clean surface the $\mathrm{Si}\left(\mathrm{L}_{23} \mathrm{VV}\right)$ transition dominated at $90 \mathrm{eV}$ energy. The $\mathrm{Au}$ $\left(\mathrm{N}_{6,7}\right.$ VV) peak, initially between two smaller silicon valence transitions, increased in magnitude with deposition and shifted to higher energy. No shift occurred for the silicon peak except at high gold coverages, for example $30 \mathrm{ML}$, when the peak was split in the manner characteristic of silicide formation (17). Figure 14 shows the variation of the magnitude of the $\mathrm{Au}\left(\mathrm{N}_{6,7} \mathrm{VV}\right)$ transition as a function of deposition time. Up to a deposition time of approximately $10 \mathrm{~s}$ there was a slow variation and thereafter an almost linear increase (also reflected in the silicon signal) until the surface was covered with approximately $1 \mathrm{ML}$ of gold. At higher coverages the intensity was proportional to $t^{2 / 3}$ and above $3 \mathrm{ML}$ there was a smooth non-linearity. The end of the linear region (approximately $1 \mathrm{ML}$ ) signifies the completion of an adlayer of gold atoms. The non-linear behaviour above $3 \mathrm{ML}$ (800 s deposition time) corresponds to the growth of small gold particles on top of a metallic layer, that is the Stranski-Krastanov

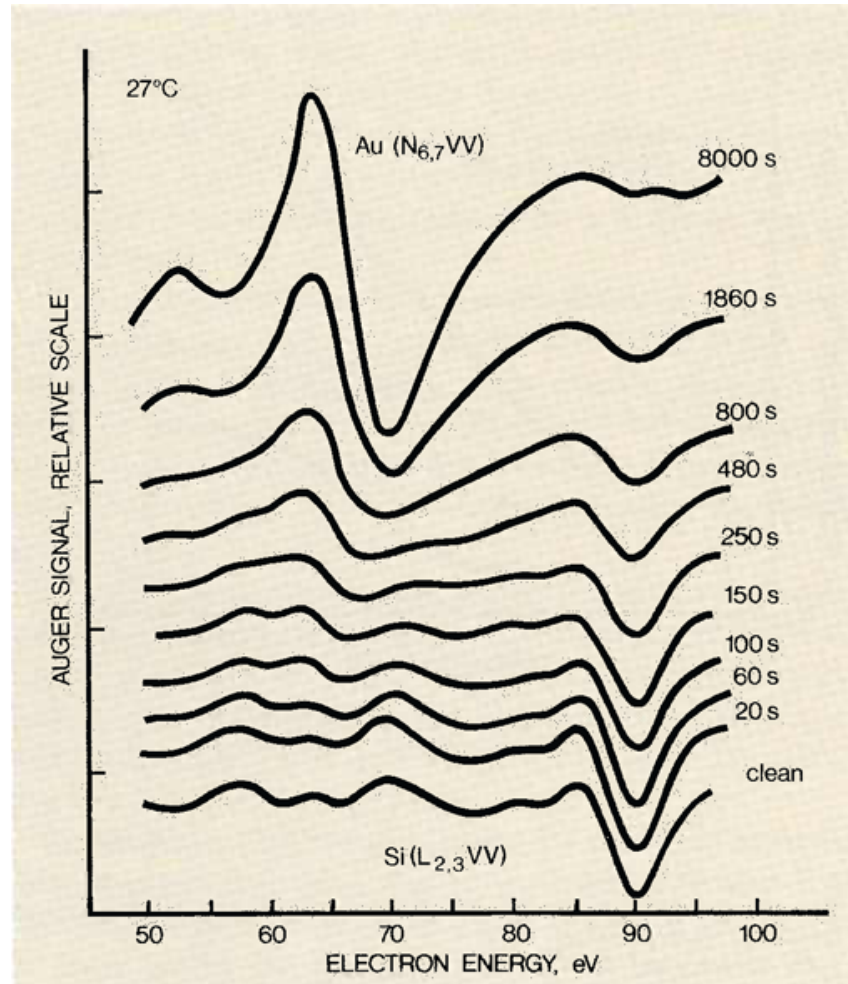

Fig. 13 Auger spectra from an oxidized surface of silicon covered by gold layers of different thicknesses represented by the deposition times

mechanism. Similar results were obtained for gold carbon (41).

The slow increase in the signal below $10 \mathrm{~s}$ represents a deficiency of metal, of the order of a few per cent, due to an initially low sticking coefficient, an inward diffusion of gold or a low probability of occurrence of the Auger transition in this highly dispersed mode. Furthermore, at such low coverages the electron bombardment could well cause surface reactions resulting in desorption and inward diffusion. The obvious conclusion drawn is that surface intermixing is low and that typically less than $10^{11}$ atoms $/ \mathrm{cm}^{2}$ diffuse into a surface layer.

The gradual change of the Auger peak energy (see the inset in Figure 14) reflects the development of gold crystallites during growth. After a deposition time of $800 \mathrm{~s}$ that energy had increased to the value for bulk gold. The increase of the Auger energy and peak broadening were consistent with an increasing crystal field resulting in a smaller binding energy and broader gold d-band (38). Thus, interaction between gold atoms started after $60 \mathrm{~s}$, that is at $0.4 \mathrm{ML}$ coverage, but the linear increase of the peak magnitude indicates essentially $2 \mathrm{D}$ clusters or domains. This was further confirmed by RHEED which at $1 \mathrm{ML}$ gave a diffuse-ring pattern. Sharp rings, due to transmission through $3 \mathrm{D}$ particles, developed at higher coverages. 


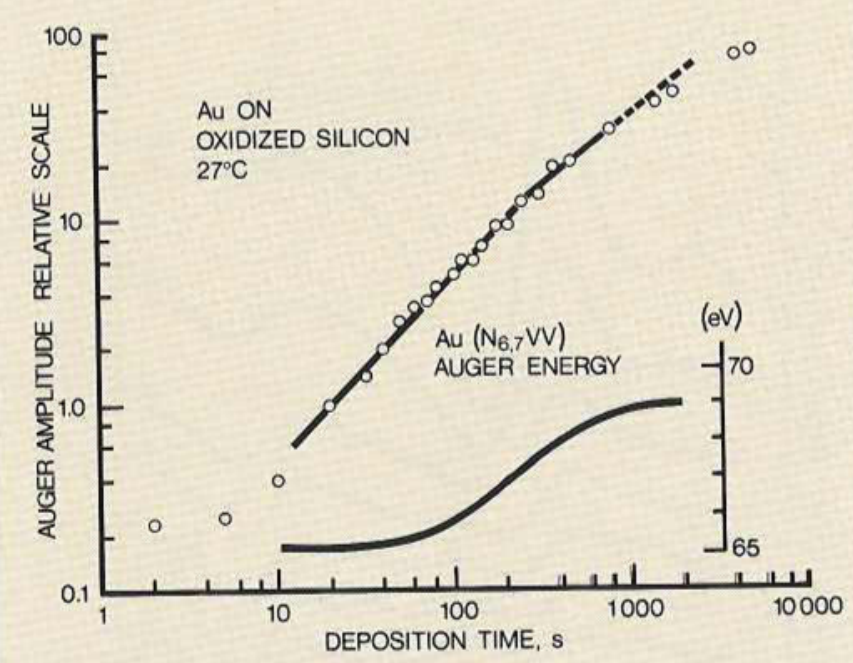

\section{References}

1 B. Lewis and J. C. Anderson, 'Nucleation and Growth of Thin Films'. Academic Press, London, 1978

2 J. C. Robins, Surf. Sat, 1979, 86, 1-13

3 G. Le Lay and R. Kern, J. Cryst. Growth, 1978, 44, 197-222

4 F. C. Frank and J. H. van der Merwe, Proc. Roy. Soc., 1949, A198, 216

5 M. D. Chinn and S. C. Fain, Jr., Phys, Rev. Lett., 1977, 39, 146-149

6 C. R. Henry, C. Chapon and B. Mutaftschiev, Thin Solid Films, 1977, $46,157-165$

7 G. Ertl and J. Küppers, 'Low Energy Electrons and Surface Chemistry'., Verlag Chemie, Weinheim, 1974

8 H. Ibach, 'Electron Spectroscopy for Surface Analysis', Springer-Verlag, Berlin, Heidelberg, New York, 1977

9 M. D. Matthews and P. F. James, Philos. Mag., 1969, 19, 1179-1188

10 H. E. Bishop and J. C. Rivière, Br.J. Appl. Phys., (J. Phys. D), 1969, 21, 1635- 1642

11 A. Hiraki, M.-A. Nicolet and J W. Mayer, Appl. Phys. Lett., 1971 _18, 178-181

12 A. Hiraki, E. Lugujjo, M.-A. Nicolet and J. W Mayer, Pbys. Status Solidi, 1971, a7, 401-406

13 A Hiraki and E. Lugujjo, J. Vac. Sci. Technol. .. 1972, 9, 155

14 A. Hiraki, E. Luguijo and J. W. Mayer, J. Appl. Phys., 1972, 43, 3643-3649

15 T. Narusawa, S. Komiya and A. Hiraki, Appl. Phys. Lett., 1972, 22, $272-273$

16 T. Narusawa, S. Komiya and A. Hiraki, Appl. Phys. Lett., 1973, 22, 389-390

17 A. Hirakı, A. Shimizu, M. Iwami, T. Narusawa and S. Komiya, Appl. Phys. Lett., 1975, 26,57-60

18 A. K. Green and E. Bauer, J. Appl. Phys., 1976, 47, 1284-1291

19 G. Le Lay and J. P. Faurie, Surf. Sci, 1977, 69, 295-300

20 L. Braicovich, C. M. Garner, P. R. Skeath, C. Y. Su, P. W. Chye, I. Lindau and W. E. Spicer, Pbys, Rev, B., 1979, 20, 5131-5141

21 K. Oura, Y. Makino and T. Hanawa, Jpn. J. Appl. Phys., 1976, 15, $737-738$

22 K. Oura and T. Hanawa, Surf. Sci., 1979, 82, 202-214

23 J. Abbati, L. Braicovich, A. Franciosi, I. Lindau, P. R. Skeath, C. Y. Su and W. E. Spicer, J. Vac. Sci. Technol., 1980, 17, 930-935

24 G. Le Lay, M. Manneville and R. Kern, Surf. Sci., 1977, 65, 261-276

25 J. O. McCaldin and T. C. McGill, in 'Thin Films Interdiffusion and Reactions', edited by J. M. Poate, K. N. Tu and J. W. Mayer, John Wiley and Sons, New York, 1978, pp. 69-80

26 A. K. Sinha and J. M. Poate, in 'Thin Films Interdiffusion and Reactions', edited by J. M Poate, K. N. Tu and J W. Mayer, John Wiley and Sons, New York, 1978, pp. 407-432
Fig. 14 Gold Auger peak-to-peak amplitude as a function of deposition time. The energy increase of the $\mathrm{Au}\left(\mathrm{N}_{6,7} \mathrm{VV}\right)$ transition is shown in the inset

\section{Conclusion}

Studies of the growth of gold films are of great significance due to their technical importance and as a means of increasing our knowledge of the growth of metal films generally. It is therefore to be expected that they will be pursued in depth in the future and extended to cover a wide range of metal/substrate combinations. Studies of the structures and compositions of the surface layers on semiconductor substrates, including studies of the effect of chemical impurities such as surface dopants, appear particularly important for better understanding both of the growth of metal films on such conductors and of the electrical behaviour of the interfaces between those films and their substrates.

27 S. P. Kowalczyk, J. R. Waldrop and R. W. Grant, Appl. Phys, Lett. $1981,38,167-169$

28 J. S. Vermaak, L. W. Snyman and F. D. Auret, J. Cryst. Growth, 1977 42, 132-135

$29 \mathrm{~K}$. Takeda, T. Hanawa and T. Shimojo, in 'Proc. 6th Internat. Vacuum Congr., 1974', Jpn. J. Appl. Phys. Suppl. 1974, 2, Pt. 1, pp. $589-592$ 30 W. Gudat and D. E. Eastman, J. Vac. Sci. Technol. 1976, 13, 831-83

31 P. W. Chye, I. Lindau, P. Pianetta, C. M. Garner, C. Y. Su and W. E. Spicer, Pbys. Rev., 1978, B18, 5545-5559

32 T. G. Andersson and S. P. Svensson, Surf Sci., 1981 submitted

33 P. Drathen, W. Ranke and K. Jakobi, Surf. Sci., 1978, 77, L162-L166

34 L. J. Brillson, R. S. Bauer, R. Z. Bachrach and G. Hansson, Appl. Phys. Lett., 1980, 36, 326-328

35 L.J. Brillson, G. Margaritondo and N. G. Stoffel, Phys. Rev. Lett., 1980 $44,667-670$

36 L. J. Brillson, G. Margatitondo, N. G. Stoffel, R. S. Bauer R. Z. Bachrach and G. Hansson, J. Vac. Sci. Technol., 1980, 17.880-885 7 T. G. Andersson, J. Appl. Phys., 1982 submitted

38 K. S. Liang, W. R. Salaneck and J. A. Aksay, Solid State Commun. $1976,19,329-334$

39 H. Takeuchi, H. Fujimoto, K. Ishii and K. Kinosta, Thin Solid Films, $1979,57,291-292$

40 M. Paunov and M. Harsdorff, Z. Naturforsch. . 1974, 29a, 1311-1318

41 R. Anton and M. Harsdorff, Thin Solid Films, 1974, 22, S23-S26 